[2] M. T. Morgan and T. A. Haley, "Design of Food Process Controls Systems,” in Handbook of Farm Dairy and Food Machinery, 2007, pp. 485-552;

[3] C. K. Martin, T. Nicklas, B. Gunturk, J. B. Correa, H. R. Allen, and C. Champagne, "Measuring food intake with digital photography,” J. Hum. Nutr. Diet., vol. 27, no. SUPPL.1, pp. 72-81, 2014;

[4] A. Yousefi-Darani, O. Paquet-Durand, V. Zettel, and B. Hitzmann, "Closed loop control system for dough fermentation based on image processing," J. Food Process Eng., vol. 41, no. 5, 2018;

[5] J. Lima, J. F. P. Moreira, and R. M. Sousa, "Remote supervision of production processes in the food industry,” in IEEE International Conference on Industrial Engineering and Engineering Management, 2016, vol. 2016-January, pp. 11231127 ;

[6] D. Kolberg and D. Zühlke, “Lean Automation enabled by Industry 4.0 Technologies,” in IFAC-PapersOnLine, 2015, vol. 48, no. 3, pp. 1870-1875.

\title{
ИДЕНТИФИКАЦИЯ МОДЕЛЕЙ ИСТЕЧЕНИЯ ЗЕРНА ИЗ ПОДСИЛОСНЫХ ЗАДВИЖЕК ДЛЯ АСОЗ ПТЛ ПЕРЕГРУЗКИ ЗЕРНА
}

\author{
Кирьязов И.Н. ${ }^{1}$, Шестопалов С.В. ${ }^{2}$, Степанов М.Т. ${ }^{3}$, Хобин В.А. ${ }^{4}$ \\ 1,2,3,4 - SE Group International, ОНАПТ (Украина) \\ E-mail: 1ivan.kiryazov@se.ua, ${ }^{2}$ stanislav.shestopalov@se.ua, ${ }^{3}$ stepanov197818@gmail.com, ${ }^{4}$ khobin@onaft.edu.ua \\ Odessa National Academy of Food Technologies, Odessa, Ukraine
}

Copyright (C) 2018 by author and the journal “Automation technologies and business - processes. This work is licensed under the Creative Commons Attribution International License (CC BY). http://creativecommons.org/licanses/by/4.0

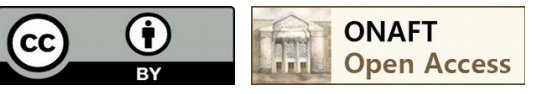

DOI: $10.15673 /$ atbp.v10i4.1232

Аннотация:Компания S-engineering входящая в холдинг SE Group International занимает лидирующие позиции в области автоматизации технологических процессов зерноперерабатывающей отрасли включая в свои проекты инновационные разработки. В частности компания занимается разработкой инновационных систем автоматической оптимизации загрузки поточно-транспортных линий (АСОЗ ПТЛ) зерновых терминалов. Разрабатываемые системы предназначены для повышения производительности ПТЛ, снижения удельных затрат электрической энергии на перегрузку, предотвращения аварийных ситуаций, связанных с завалами зерна в башмаках норий и надвесовом бункере весов из-за перегрузки ПТЛ, Указанные задачи решаются системой в условиях формирования потока зерна одновременно из нескольких источников. ACO3 реализует технологии Leffol \& Senuтас $(L \& S)$, запатентованные SE Group International. Технология LEFFOL: способ контроля степени загрузки конвейера / Method of Loading Efficiency Control. Технология SENUMAC: способ автоматического управления загрузкой поточно-

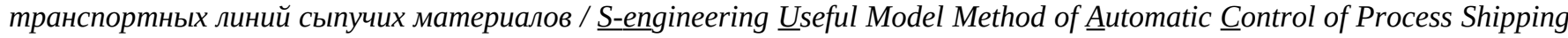
line upload of granular materials.

Разработка таких систем включает несколько этапов, одним из которых является этап исследования функционирования ПТЛ, изучение их свойств как объектов управления с целью дальнейшего отражения этих свойств в алгоритмах управления. Особое внимание уделяется изучению подсилосных задвижек, так как их конструкция, размеры, принцип установки значительно влияют на процесс истечения зерна.

В данной статье рассматривается экспериментальное получение математических моделей истечения зерна из подсилосных задвижек для дальнейшей их реализации в алгоритмах управления АСОЗ ПТЛ перегрузки зерна на суда.

Abstract: S-engineering company, which is part of the holding company SE Group International, occupies a leading 
position in the field of automation of technological processes in the grain processing industry, including innovative developments in its projects. In particular, the company is engaged in the development of innovative automated loading optimization system of the flow-transport lines (ALOS FTL) of grain terminals. The developed systems are designed to improve the performance of FTL, reduce the unit cost of electrical energy for overload, prevent accidents associated with grain blockages in the elevator shoes and overhang hopper of the scales due to FTL overload. These problems are solved by the system in the conditions of the formation of grain flow simultaneously from several sources. ALOS implements Leffol \& Senumac (L\&S) technology, patented by SE Group International. LEFFOL technology: method of controlling the degree of loading of the conveyor / Method of Loading Efficiency Control. SENUMAC technology: a way to automatically control of

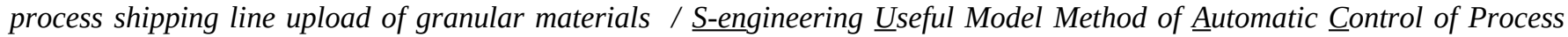
Shipping line upload of granular materials.

The development of such systems includes several stages, one of which is the stage of studying the functioning of FTL, the study of their properties as control objects with a view to further reflect these properties in control algorithms. Particular attention is paid to the study of the silo gates, as their design, size, installation principle significantly affect the process of the outflow of grain.

The article deals with the experimental obtaining of mathematical models of the outflow of grain from the silo gates for their further implementation in the control algorithms of the ALOS FTL for loading grain onto ships.

Ключевые слова: перегрузка зерна, поточно-транспортные линии, производительность, энергозатраты, автоматизированная система оптимизации загрузки.

Keywords: grain reloading, flow-transport lines, productivity, energy consumption, automated loading optimization system.

Постановка задачи.

АСОЗ ПТЛ перегрузки зерна [1-3], внедренная на нескольких зерновых терминалах является инновационной разработкой компании S-engineering. Ее цель - формирование потока зерна одновременно из нескольких источников, повышение производительности ПТЛ, снижение энергозатрат на перегрузку, предотвращение аварийных ситуаций, связанных с завалами зерна в башмаках норий и надвесовом бункере весов из-за перегрузки ПТЛ. АСОЗ реализует технологии Leffol \& Senumac (L\&S), запатентованные SE Group International [4-5].

Функции, реализуемые АСОЗ.

1. Автоматический расчет степени открытия задвижек по заданному соотношению расходов зерна из заданных силосов в его общий поток на отгрузку.

2. Автоматическая стабилизация производительности общего потока зерна (загрузки ПТЛ).

3. Автоматическое предотвращение аварийных остановов ПТЛ из-за развития завалов зерна в башмаках норий и надвесовом бункере весов (при ограничении производительности весов установленным положением задвижки подвесового бункера).

4. Автоматический вывод производительности ПТЛ на максимально достижимую в текущих условиях величину.

5. Автоматическое быстрое (после команды оператора, вызванной необходимостью изменить режим загрузки судна) изменение производительности ПТЛ до одного из двух заданных сниженных уровней и возврат в режим ее оптимизации.

Для реализации эффективного управления в алгоритмах ACO3 в форме математеческих моделей отражаются особенности процесса перегрузки зерна как объекта управления. В частности, реализуются математические модели процесса истечения зерна из подсилосных задвижек. Характер истечения зерна определяется его видом, механической конструкцией выпускного устройства, расположением задвижек с ручным и от двигателя перемещением шибера и рядом других факторов индивидуальных для каждого элеватора. В таких условиях наиболее конструктивным является комбинированный или сугубо экспериментальный методы получения математической модели процесса истечения зерна.

\section{Методы и материалы исследований}

Для получения математических моделей процесса истечения зерна из подсилосных задвижек на элеваторе «Новотех-терминал» (г.Одесса) были проведены ряд активных экспериментов по получению расходных характеристик задвижек. На рис.1 в качестве примера представлен вид окна с диаграммами изменения производительности ПТЛ отгрузки зерна в судно и степени открытия подсилосной задвижки при проведении активного эксперимента по снятию динамических характеристик и расходной характеристики подсилосной задвижки. А в таблице №1 сведены экспериментальные данные, полученные при определении расходной характеристики подсилосной задвижки силоса "С3-3" элеватора "Новотех-терминал" для зерна рапса. 


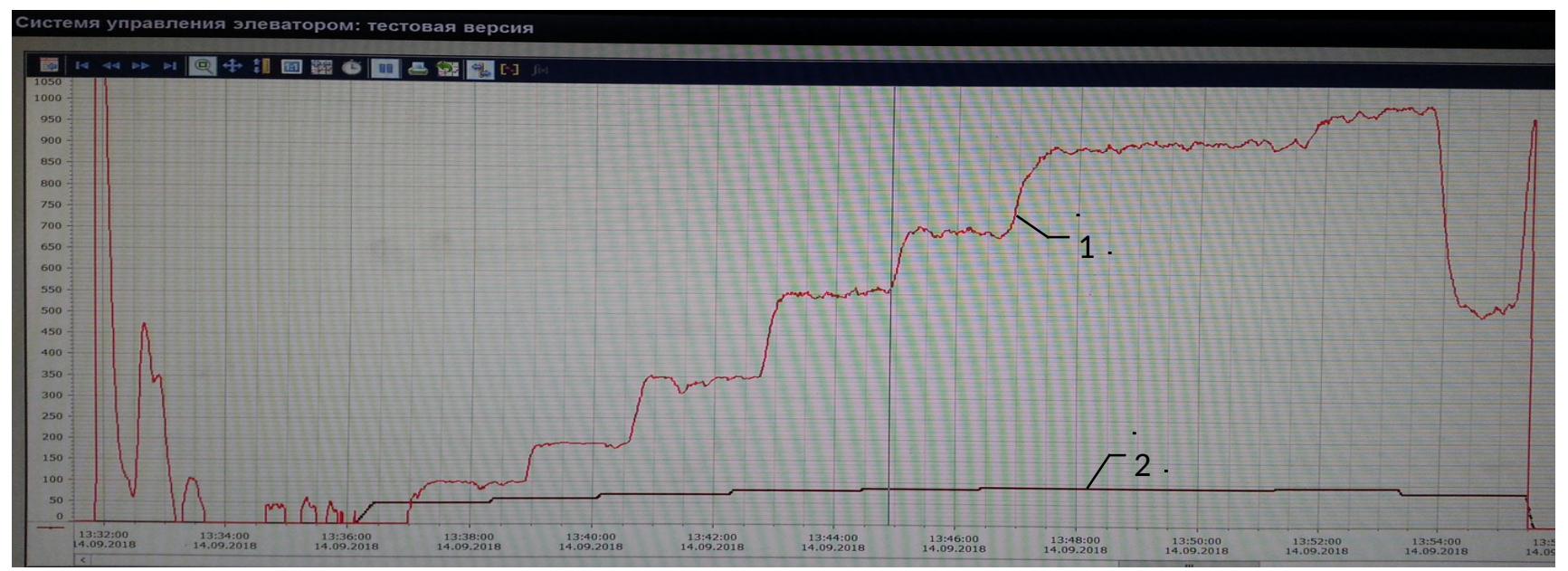

Рис. 1 - Вид окна с диаграммами изменения производительности 1 (т/ч) ПТЛ отгрузки зерна в судно и степени открытия подсилосной задвижки 2 (\%) при проведении активного эксперимента по снятию расходной характеристики подсилосной задвижки

Таблица 1 - Экспериментальные данные, полученные при определении расходной характеристики подсилосной задвижки силоса "С3-3" элеватора "Новотех-терминал" для зерна рапса

\begin{tabular}{|c|c|c|c|}
\hline № эксперимента & $\begin{array}{c}\text { Степень открытия } \\
\text { подсилосной } \\
\text { задвижки, } \%\end{array}$ & $\begin{array}{c}\text { Производительность } \\
\text { ПТЛ (расчетная), } \\
\text { т/ч }\end{array}$ & $\begin{array}{c}\text { Производительность } \\
\text { ПтЛ (показания } \\
\text { бункерных весов), } \\
\text { т/ч }\end{array}$ \\
\hline 1 & 50 & 100 & 108 \\
\hline 2 & 60 & 185 & 175 \\
\hline 3 & 70 & 345 & 344 \\
\hline 4 & 80 & 550 & 584 \\
\hline 5 & 85 & 705 & 716 \\
\hline 6 & 90 & 910 & 950 \\
\hline 7 & 92 & 1000 & 1050 \\
\hline
\end{tabular}

Идентификация моделей выполнялась в программе «Curve Fitting Tool» системы MATLAB. При проведении идентификации моделей учитывалась физическая сущность процесса истечения зерна, необходимая точность модели и ее простота для дальнейшей реализации в алгоритмах АСОЗ ПТЛ. Поэтому в качестве структуры модели был выбран полиномом второго порядка. На рис.2 представлены Результаты идентификации модели расходной характеристики подсилосной задвижки в программе «Curve Fitting Tool». В структуре модели отсутствуют физически не обоснованные перегибы функции. График модели достаточно точно соответствует экспериментальным данным, что подтверждается полученными значениями числовых коэффициентов, определяющих точность соответствия модели экспериментальным данным.

\section{Результаты исследований.}

Полученная модель отражает значительную нелинейность расходной характеристики подсилосной задвижки. Учет этой нелинейности позволит правильно формировать расход зерна из каждой задвижки при формировании потока зерна из нескольких силосов одновременно. Дополнительно, полученная модель отражает значительное изменение коэффициента передачи в канале управления на разных производительностях. При изменении заданной производительности с 200т/ч до 950 т/ч коэффициент передачи будет меняться примерно в 5 раз. Такие значительные изменения свойств канала управления должны быть отражены в алгоритмах управления и обеспечить высокий запас устойчивости системы. 

http://www.atbp.onaft.edu.ua/

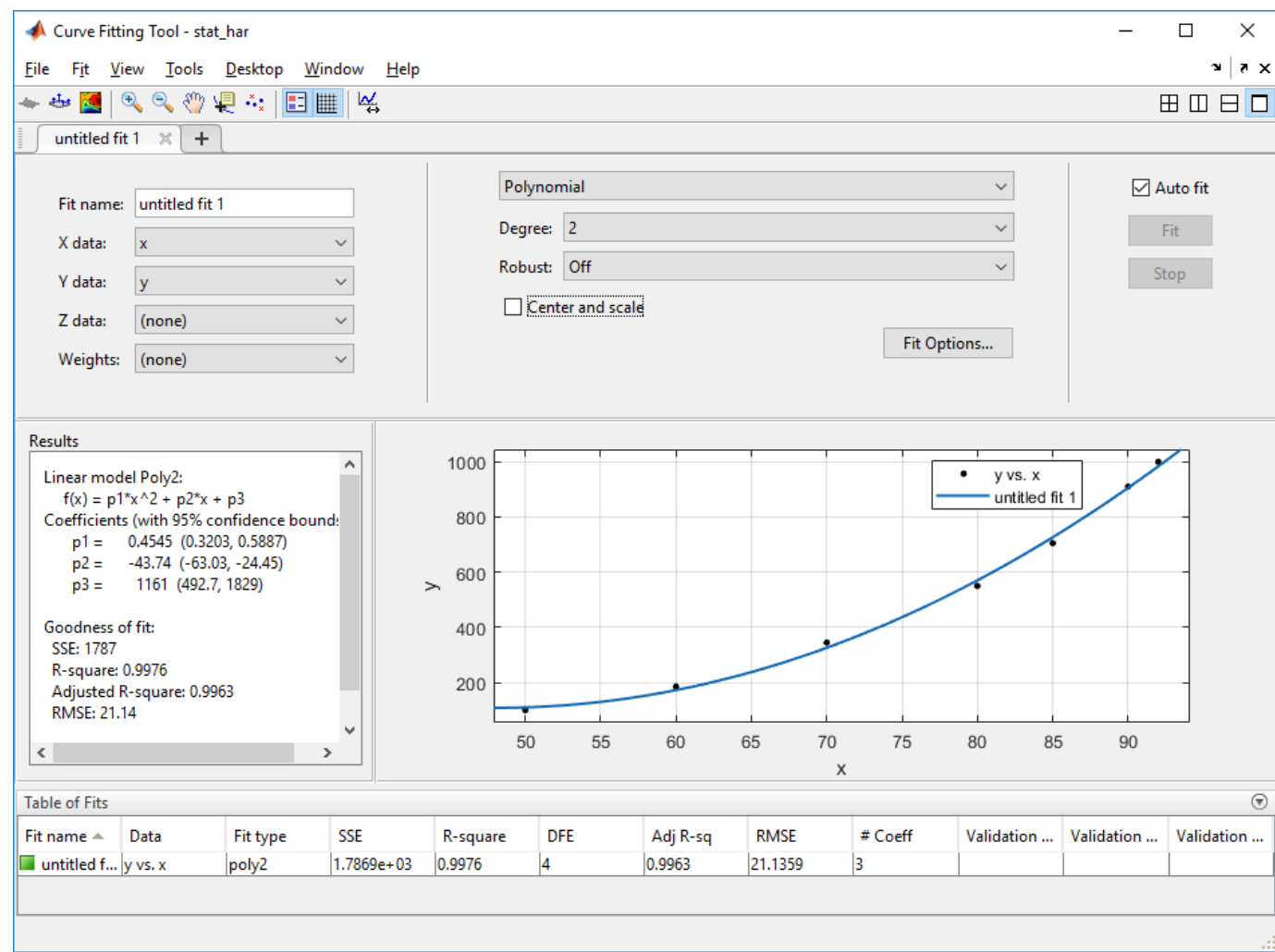

Рис. 2 - Результаты идентификации модели расходной характеристики подсилосной задвижки в программе «Curve Fitting Tool» системы MATLAB на основе экспериментальных данных, полученных при отгрузке рапса на судно из силоса "СЗ-3" элеватора "Новотех-терминал"

\section{Выводы.}

В результате проведенных исследований получена модель истечения зерна рапса из подсилосных задвижек элеватора "Новотех-терминал" (г. Одесса). Модель может быть отмасштабирована и применяться к другим видам зерна. Учет свойств модели в алгоритмах управления АСОЗ ПТЛ отгрузки зерна позволит обеспечить быстрый вывод ПТЛ на заданную производительность, высокую точность процентного ввода зерна при его отгрузке из нескольких силосов одновременно, стабильную и устойчивую работу системы в различных режимах.

\section{Список использованных источников}

[1] Хобин В. А. Концепция эффективного управления производительностью поточно-транспортных линий при перегрузке зерна / В. А. Хобин, С. В. Шестопалов // Хранение и переработка зерна. - Днепропетровск, 2011. № 10 (148). - С. 26-29;

[2] Шестопалов С. В. Оптимизация производительности процесса перегрузки зерна в условиях ограничения типа «аварийная ситуация» / С. В. Шестопалов, В. А. Хобин // XVIII Міжнарод. конф. 3 автомат. управління «Автоматика - 2011». - Львів, 2011. - С. 194-195;

[3] Кирьязов И. Н. Автоматизированная система оптимизации загрузки поточно-транспортных линий перегрузки зерна: итоги производственных испытаний / И. Н. Кирьязов, С. В. Шестопалов // Хранение и переработка зерна. - Днепропетровск, 2013. - № 7 (172). - С. 43-46.

[4] Пат. на винахід 99525 Україна, МПК (2011.01), B65G 17/00, G01R 29/00. Спосіб контролю ступеня завантаження конвеєра / Аннаев Б. С., Герасимов В. В., Хобин В. А., Кирьязов И.Н., Шестопалов С. В. и др.; власник ТОВ «С-Інжинірінг». - № а201014455; заявл. 03.12.10; опубл. 25.05.12, Бюл. № 10. - 14 с.

[5] Пат. на винахід 95887 Україна, МПК (2011.01), B65G 17/00, B65G 47/46 (2006.01), B65G 65/42 (2006.01), G01G 11/12 (2006.01). Спосіб автоматичного управління завантаженням потоково-транспортної лінії сипких матеріалів / Аннаєв Б. С., Герасимов В. В., Хобін В. А., Кір’язов І. М., Шестопалов С. В. і ін.; власник ТОВ «СІнжинірінг». - № а201015861; заявл. 29.12.10; опубл. 12.09.11, Бюл. № 17. - 24 с;

\section{References}

[1] V. A. Khobin et al. "Kontseptsiya effektivnogo upravleniya proizvoditelnostyu potochno-transportnyih liniy pri peregruzke zerna”, Hranenie i pererabotka zerna, vol. 10, pp. 26-29, 2011. 
[2] S. V. Shestopalov et al. "Optimizatsiya proizvoditelnosti protsessa peregruzki zerna v usloviyah ogranicheniya tipa «avariynaya situatsiya”, XVIII Mizhnarod. konf. z avtomat. upravlinnya «Avtomatika-2011», Lviv, UA, 2011. pp. 194-195.

[3] I. N. Kiryazov et al. “Avtomatizirovannaya sistema optimizatsii zagruzki potochno-transportnyih liniy peregruzki zerna: itogi proizvodstvennyih ispyitaniy”, Hranenie i pererabotka zerna. vol. 7, pp. 43-46, 2013.

[4] B. S. Annaev et al. “Sposib kontrolu zavantazhennya konveera”, UA Patent 99525, May, 25, 2012.

[5] B. S. Annaev et al. "Sposib avtomatichnogo upravlInnya zavantazhennyam potokovo-transportnoyi liniyi sipkih materialiv”, UA Patent 95887, September, 12, 2011.

\title{
ОПТИМИЗАЦИЯ ПРОЦЕССА АВТОМАТИЧЕСКОЙ СИНХРОНИЗАЦИИ СУДОВЫХ ДИЗЕЛЬ-ГЕНЕРАТОРОВ ПРИ ДЕТЕРМИНИРОВАННОЙ ПОСТАНОВКЕ ЗАДАЧИ
}

\author{
Шевченко В.А. \\ Национальный университет «Одесская морская академия», Одесса, Украина \\ ORCID: 0000-0003-3229-1909 \\ E-mail: vash4891@gmail.com
}

Copyright (C) 2018 by author and the journal “Automation technologies and business - processes. This work is licensed under the Creative Commons Attribution International License (CC BY). http://creativecommons.org/licanses/by/4.0
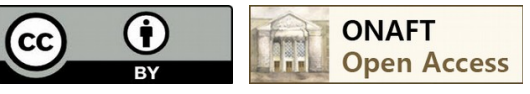

DOI: $10.15673 /$ atbp.v10i4.1233

Аннотация:Процесс управления синхронизацией генераторов является одним из наиболее сложных процессов в судовых электроэнергетических установках. Разработке методов абстрактного и структурного синтеза устройств автоматической синхронизации с применением последних достижений электронной промышленности, а также синтезу алгоритмического обеспечения для программируемых систем уделяется недостаточно внимания. В работе предложено математическое и алгоритмическое описание процесса автоматической синхронизации дизельгенераторов в судовой электроэнергетической установке. Выделены три вида критериев оптимальности управления процессом синхронизации при детерминированной и стохастической постановке задачи. Определены риски отклонения фактической траектории синхронизируемого объекта от ожидаемой. Получена обобщенная структура управления процессом синхронизации и алгоритм функционирования системы управления этим процессом. Определена структурная схема канала подгонки частоты синхронизируемого дизель-генератора, а также описаны передаточные функции каждого ее звена. Определены математические выражения времени ожидания наступления момента синхронизма, а также параметров, определяющих задержку времени срабатывания генераторного автомата. Получена диаграмма, иллюстрирующая способ определения параметров синхронизации. Наглядное описание процесса при помощи алгоритмов, диаграмм и математических выражений позволит без труда реализовать предложенный способ в современных системах автоматического управления судовыми электроэнергетическими установками.

Abstract:Generators synchronization control process is one of the most complicate processes of the ship's electrical power plant. Automatic synchronization control devices development abstract and structure synthesis methods, programmable synchronization systems algorithms receives little attention. Diesel-generator automatic synchronization process mathematical and algorithmic description is proposed in the paper. It is selected three optimization criterions on synchronization process control in deterministic and stochastic task formulation. Synchronization object actual trajectory deviation from expected trajectory risks were defined. Synchronization control process generalized structure and such process control system algorithm were received. Synchronizing diesel-generator speed control channel structure diagram and it's all transfer functions were defined. Synchronism moment expectation time and generator's circuit breaker closing time delay mathematical expressions were defined. Synchronization parameters defining diagram is presented in paper. Synchronization 